\title{
High energy physics and very high energy astrophysics
}

\author{
Lawrence W. Jones ${ }^{\text {a }}$ \\ University of Michigan, Ann Arbor, Michigan, USA
}

\begin{abstract}
The current research programs at the CERN LHC and at the U.S. Fermi National Accelerator Laboratory (FNAL) and other activities are briefly reviewed. The programs which are related to cosmic ray issues and which form a link between the cosmic ray community and the particle accelerator community are noted. The AMS-02 satellite program is also noted.
\end{abstract}

\section{Introduction}

What do we know and what are our problems? Recently, the Higgs Boson was discovered at CERN, with global recognition and enthusiasm. The "Standard Model" of elementary particle physics is now well established, and (at least by theorists) well understood. Gravity Waves have now been observed by LIGO, the Laser Interferometer Gravity Wave Observatory (actually, two detectors; one in the State of Washington, and the other in the State of Louisiana). The neutrino is now understood to have 3 "flavors" and 3 different masses and the neutrino oscillates between these states. Dark matter remains a puzzle. A dark matter substance is required to understand the orbits of stars in galaxies, and also even the bending of light rays; e.g. light from distant astronomical objects passing close to a galaxy. There is currently no clue concerning the nature of dark matter. A popular guess is that it is composed of a flux of massive and very weakly interacting elementary particles, but to date there has not been any observed evidence for such particles. The question of Dark Energy is even more puzzling. The galaxies in the universe appear to be flying apart - accelerating - as if some force were driving them, however we have no idea what this force could be. A more relevant question in this Symposium is the source of cosmic rays; where do they come from, and what accelerates them? To be sure, explosions, collisions, or other unusual phenomena at galactic centers are good candidates, but much has yet to be learned.

It turns out that there have been several relevant conferences and meetings recently in the U.S. and in Europe. At the beginning of August there was a meeting in Chicago; "ICHEP 2016", the 38th International Conference on High Energy Physics. There was also a meeting in Michigan recently; "Cosmo-16", with much discussion of topics like dark matter and dark energy. In June 2016, there was the annual meeting of the Fermilab (FNAL) Users Organization, and the lectures there were a good presentation of the current activities at Fermilab. A recent issue of the CERN periodical, the "CERN Courier", contains useful reviews of the research programs of the four major experimental programs at the LHC (Large Hadron Collider); CMS, ATLAS, LHCb, and ALICE.

a e-mail: 1wjones@umich.edu

\section{FERMILAB and CERN, current activities and projects}

Since the Tevatron (the $2 \mathrm{TeV}$ c.m. anti-proton, proton collider) was terminated (following some decades of excellent research), Fermilab has focused its activities on muon and neutrino physics. Indeed, physicists are still producing good physics from the extensive data collected at the Tevatron. Also, many Fermilab physicists are active in CERN LHC experiments, in particular CMS, which has a major analysis program and analysis facility at Fermilab. Members of the Fermilab staff are also active in cosmic ray programs, such as the Pierre Auger experiment in Argentina, as well as in searches for Dark Matter and Dark Energy.

Although managed and financed by the U.S. Department of Energy, Fermilab also has many physicists from other countries involved in its activities. One program which includes a significant Russian involvement is related to the SeaQuest experiment. This employs a proton beam of $120 \mathrm{GeV}$ and uses the Drell-Yan process to probe the anti-quark structure of target nucleons. The asymmetry between anti-down quarks and anti-up quarks in the nucleon sea is measured in targets of liquid hydrogen and liquid deuterium, as well as other target materials.

One project with this facility is to produce a polarized proton beam to study spin effects. However, the generation of a polarized beam at high energies is a problem. A proton beam at low energy can be polarized easily, but in a multi$\mathrm{GeV}$ circular accelerator, maintaining the polarization is a problem. When the protons circulate around an alternating gradient ring thousands of times, the polarization is lost.

In 1978, Ya.S. Derbenev and A.M. Kondratenko (then at Novosibirsk) conceived of a way to preserve the polarization. The solution is to rotate the spin of the protons 180 degrees twice per revolution. Note that, in a horizontal accelerator, the spins are aligned vertically, parallel to the dominant magnetic field. Derbenev argued that the rotation of the spins could be accomplished by a toroidal magnet, and with these magnets and the spin rotations, the spins were retained during acceleration. Ernest Courant labeled these magnets "Siberian Snakes".

Besides the work at Michigan relevant to polarized beams for SeaQuest, a Russian group is also working on 
having a polarized proton beam in the NICA accelerator at Dubna. This group includes A.S. Belov, Kovalenko, A.M. Kondratenko, Yu Filatov, V.S. Morozov, M.N. Ukhanov, and Troshin. The Michigan group is headed by Alan Krisch, and the Michigan and Russian groups remain in close contact (including monthly international discussions) on topics and problems related to polarized beams. Although these programs are not related to cosmic ray physics, because of the close Michigan - Russian liaison and physics objectives, they merit recognition at this international conference.

\subsection{CERN LHC program}

The CERN Large Hadron Collider is colliding proton beams of $7.5 \mathrm{TeV}$, producing collisions of $13 \mathrm{TeV}$ (centerof-mass), equivalent to cosmic ray proton energies of about $100 \mathrm{PeV}$ colliding with stationary protons. Four major experiments are in operation there.

CMS is the largest of the LHC experiments. This collaboration is seeking evidence for more massive versions of the $\mathrm{W}$ and $\mathrm{Z}$ bosons, as well as heavier, integral-spin versions of the six known quarks.

$\mathrm{LHCb}$ has been an experiment studying b-quarks and other heavy quarks in greater detail. Although a further upgrade of this detector is planned, they will continue their studies in greater detail and complexity with the up-graded LHC.

ATLAS is also a very large detector, like CMS, which observes close to $4 \pi$ steradians final-state solid angle (as does CMS). One of its current objectives is the search for evidence of Dark Matter. Astronomical observations confirm the existence of Dark Matter, but we have no idea of its nature. Assuming that Dark Matter is composed of weakly-interacting particles with a rest mass accessible at the LHC, the ATLAS data enables searches for evidence of their production.

ALICE is a detector with a primary objective of studying proton - lead $(\mathrm{p}-\mathrm{Pb})$ collisions, and comparing them with proton - proton $(p-p)$ collisions, in the distribution of reaction products, particle jets, etc. As with $\mathrm{LHCb}$, the ALICE detector array subtends a smaller solid angle than the ATLAS and CMS detectors, but its results are relevant and interesting.

At the meeting of this Symposium at CERN in 2014, I noted that a useful LHC experiment would be the study of collision of protons with oxygen, nitrogen, or carbon nuclei, to replicate the interactions of proton cosmic rays in the air. Such data would be very useful for the cosmic ray community in better understanding proton-initiated air showers, to aid in the interpretation of air shower data from ground-level detectors (e.g. the distribution and density of gamma ray, electron, and muon distributions). Unfortunately, none of the LHC groups has undertaken this study, but it would still merit the efforts of the cosmic ray community to encourage such a study by one of the LHC groups. Such data, at a modest requirement of running time and effort by an LHC program (e.g. ATLAS or CMS) would be very valuable for the cosmic ray community in their use of air shower data in programs such as AUGER.

\section{Muon physics}

An area of physics which Fermilab is now pursuing is the study of muons. Protons accelerated to about
$120 \mathrm{GeV}$ produce pions in their interaction in a static target; these pions, with a mean lifetime of about 28 nano-seconds, decay in flight into muons, with a lifetime of about 2.2 microseconds, and the resulting muon flux can be contained, controlled, and used. For example, Fermilab currently has a program to make a more accurate measurement of "G-2" (the Gyromagnetic ratio of the muon minus 2.000). While this has been measured at Brookhaven as well as at CERN, starting in the 1960s, a more accurate measurement will be possible at Fermilab. The Brookhaven storage-ring permanent magnet has been shipped to Fermilab, and will be used - as it was at Brookhaven - for these measurements.

Among other possible muon physics, there is also discussion about the possibility of building a "muon collider"; positive and negative muons would be collected and injected into a storage ring, circulating in opposite directions, and the properties of their interactions would be studied. As with electron - positron colliders, interesting heavy mesons would be produced and studied.

\section{Neutrino physics}

From recent studies, it is known that there are 3 "flavors" of neutrinos; Electron neutrinos, Muon neutrinos, and Tau neutrinos. And there are also 3 neutrino masses (each less than about 2 electron volts), the mass states and the flavor states are not the same. Neutrinos oscillate between their different modes, e.g. the neutrinos from nuclear interactions in the Sun are electron neutrinos, but when studied at the Earth, only about a third of the Solar neutrino flux are electron neutrinos; comparable numbers of muon neutrinos and tau neutrinos are also observed. Understanding this complex situation of the relationship between the three mass states and the three flavor states, is unusual and complex. It is the goal of current neutrino experiments to understand and explain this unusual situation.

There are two neutrino experiments which have Fermilab as their neutrino source. NOvA is a Fermilab neutrino experiment in which muon neutrinos are generated by pion decays, which are produced by the $120 \mathrm{GeV}$ proton beam, pass through a 300 ton detector on the Fermilab site and are directed toward a 14,000 ton detector in a mine located in northern Minnesota, 810 kilometers distant. The detectors utilize $4 \times 6 \mathrm{~cm}$ liquid scintillator-filled PVC cells as their detectors. While the detector on the Fermilab site detects almost 100\% muon neutrinos, those detected in Minnesota are a mixture of the three different flavors. The data from NOvA are enabling the knowledge of the matrices relating the massive and flavor neutrinos.

Another Fermilab neutrino experiment is DUNE (Deep Underground Neutrino Experiment) at the Sanford site in South Dakota. This experiment, still under construction at the Sanford site, uses four liquid Argon detectors, each containing 10,000 tons of liquid argon. The liquid Argon serves as a detector for the Cherenkov radiation produced by muons and electrons from neutrino interactions. As with NOvA, DUNE is located deep underground to minimize the flux of cosmic ray muons (and other particles). The Sanford site is a former gold mine, about $1.5 \mathrm{~km}$ deep, and is located $1300 \mathrm{~km}$ from Fermilab. At Fermilab, as with NOvA, the neutrinos are generated from 
pion decays, with a high flux of pions produced by the interactions of $120 \mathrm{GeV}$ protons. There will be 4 similar but smaller - liquid argon detectors on the Fermilab site. The DUNE program is an international collaboration with many participants from Europe and elsewhere.

Although unrelated to Fermilab, it is worth noting here the underwater neutrino experiments, which have a rich history. All neutrino experiments require an enormous volume of material, because of the very small interaction cross section of the neutrinos. So for many years, neutrino experiments have comprised of detector arrays deployed deep under water, in lakes and/or seas. The detectors (usually phototube arrays) observe the Cherenkov radiation produced by electrons or muons, which in turn are generated by neutrino interactions. In the last century, DUMAND was a concept for a neutrino detection experiment to be located in the Pacific Ocean, off the coast of Hawaii. It never materialized, regrettably. ANTARES was located in the Mediterranian Sea, as is KM3NET (still under construction). Lake Baikal is the home to Baikal-GVD (where GVD stands for "Gigaton Volume Detector").

A successor to the deep under-water neutrino detectors is IceCube, a cubic kilometer array of photodetectors in the Antarctic ice (near the South Pole). The detector is a matrix of 5160 photo-detectors, each of which is a spherical module containing several photomultipliers. These are deployed within the cubic kilometer of ice, which is $1.5-2.5 \mathrm{~km}$ below the surface. While neutrinos from cosmic rays interacting in the Earth's atmosphere are observed, the primary objective is to observe cosmic neutrinos which have come directly from their sources; the only component of cosmic ray particles which come directly from their sources (e.g. not deflected by galactic magnetic fields or obstructed by nuclear interactions). Since 2012, IceCube has observed 54 neutrino events (via muons and electron showers) from 30 to $2000 \mathrm{TeV}$.

One neutrino phenomenon reported some years ago, from a neutrino experiment at Los Alamos, was the possible existence of a "Sterile Neutrino", e.g. a neutrino which did not have a flavor like the three known neutrinos, and did not transform. Other experiments have sought evidence for these Sterile Neutrinos but none have seen evidence for it. The IceCube experiment has recently reported strong negative evidence for such a neutrino.

\section{Dark matter}

It is certainly true that a major mystery in particle physics and cosmology currently is the nature of Dark Matter. The existence of Dark Matter comes from the observations of the stellar dynamics of galaxies; the fact that the orbital velocities of stars in the outer reaches of a galaxy require a larger galactic mass than is observed. Also, the bending of light (from more distant objects) passing a galaxy requires more than the visible mass. This missing mass can be most easily explained as due to a population of massive, but weakly-interacting particles. As weakly-interacting particles, without strong or electromagnetic interactions (like neutrinos), they would impact galactic dynamics but would otherwise have no observable properties. However, if they had weak interactions (like neutrinos), they should rarely induce an observable particle interaction, hence this is the basis for current searches by particle physicists.

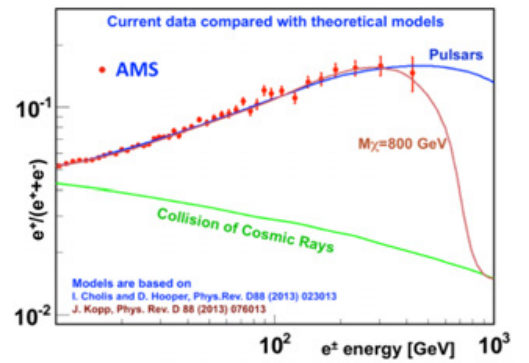

Figure 1. Positron fraction, $e^{+} /\left(e^{-}+e^{+}\right)$, of the electron plus positron flux between 10 and $1000 \mathrm{GeV}$.

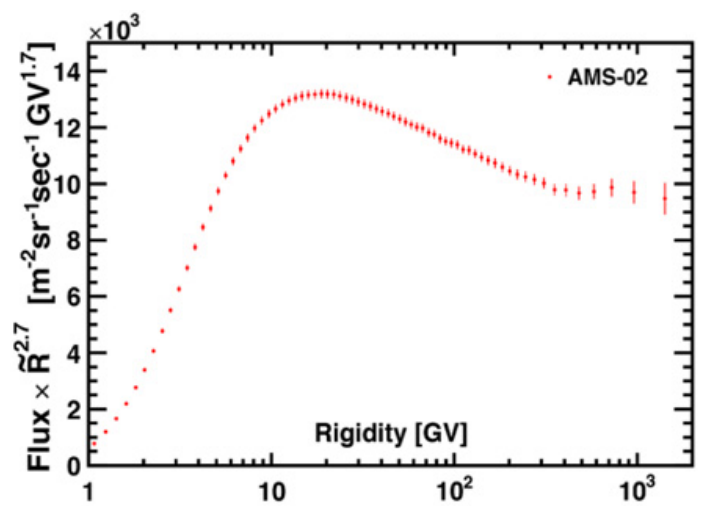

Figure 2. Precision measurement of the proton flux in primary cosmic rays from rigidity $1 \mathrm{GeV}$ to $1.8 \mathrm{TeV}$, based on 300 million events.

There have been and are currently many searches for evidence of weakly-interacting paricles which could be the substance of Dark Matter. Fermilab physicists have a major Dark Matter search program in progress, using a very large detector in the South Dakota Sanford mine. Named LUX (for Liquid Underground Xenon), it is a $370 \mathrm{~kg}$ volume of liquid Xenon, and located at $1480 \mathrm{~m}$ underground in the Sanford mine. Operating since 2012, the search for WIMPs (Weakly Interacting Massive Particles) has come up empty.

Of course, other similar searches are in progress at other sites, and by other groups. Dark Matter remains one of the current three major mysteries in the physics of Cosmology-Astrophysics; the other two are the nature of Dark Energy (the source of the continued accelerationexpansion of the Universe) and the details of the evolution of the early Universe (its minute size, dramatic explosion, etc.).

\section{AMS-02}

One interesting program not otherwise included among the presentations at this Symposium is a report on the AMS-02 satellite experiment aboard the International Space Station. This is a major program directed by Professor Samuel Ting of M.I.T. Ting's original objective was to explore whether heavy nuclei (high atomic number, A) had equal matter and antimatter components. Observations of electromagnetic radiation (light, X-rays, infra-red, radio, etc.) are insensitive to whether their source is matter or antimatter. Ting argued that, since galaxies are separate and spaced apart, it would be possible that perhaps half of the galaxies are composed of anti-matter, and therefore, observing energetic nuclear cosmic rays, identifying 


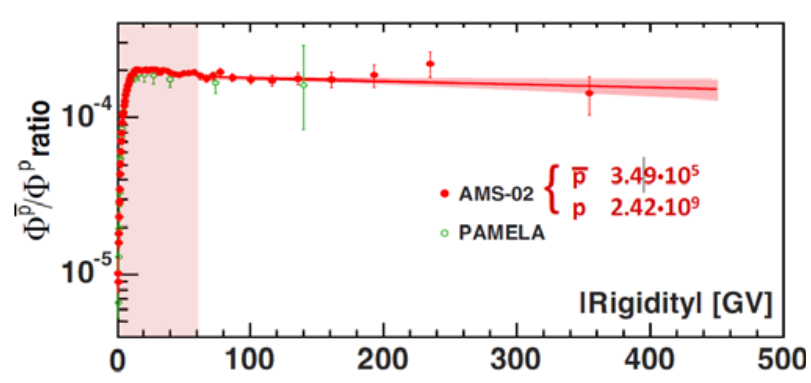

Figure 3. Flux ratio of anti-protons to protons up to $400 \mathrm{GeV}$.

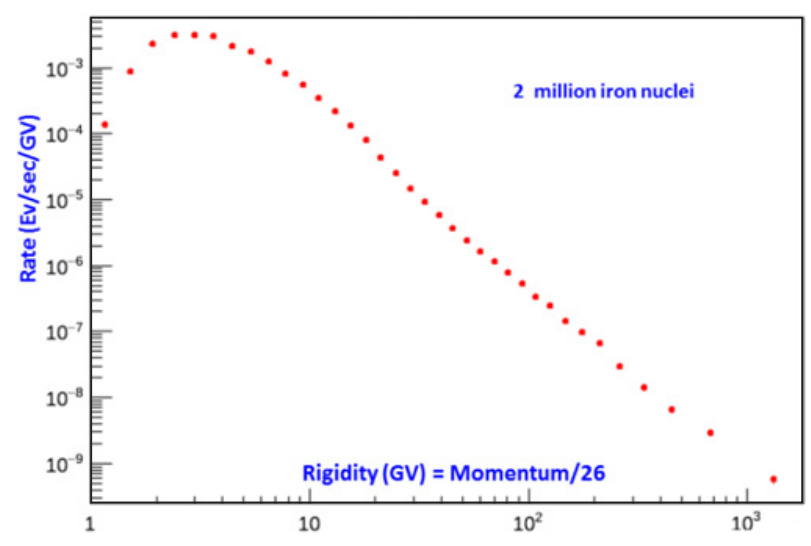

Figure 4. Flux of 2 million iron nuclei measured by AMS vs. energy (momentum) divided by the nuclear charge, $Z=26$ between $1 \mathrm{GeV}$ and $1 \mathrm{TeV}$.

their electric charge and mass, would enable the search for primordial antimatter. The community of traditional cosmic ray physicists were skeptical of the idea that half of the universe would be antimatter, arguing that the galaxies were not so far separated that there wouldn't be evidence for matter-antimatter annihilation between galaxies. Such annihilation would be observable, e.g. as sources of energetic gamma radiation, etc. To be sure, the matter anti-matter asymmetry of the Universe remains a problem.

But a cosmic ray magnetic spectrometer, designed to study the mass, energy, and electric charge of cosmic ray nuclei, would be a useful tool for better understanding the nature of primary cosmic rays, therefore NASA (National Aeronautics and Space Administration, in America) approved Ting's concept.

He then set out to build this spectrometer, which would be located on the International Space Station, a large satellite program that was already in orbit. Ting labelled his detector the "Anti-Matter Spectrometer", or AMS. However NASA did not like a U.S. space program to employ "Anti" in its name, so Ting's program was renamed the "Alpha Magnetic Spectrometer" (hence still AMS). The detector is a magnetic spectrometer, using a permanent magnet. Originally, Ting had considered a super-conducting magnet, however the desire to collect data for several years made an electro-magnet impractical. Ting's group built a prototype magnetic spectrometer, AMS-01, which was flown earlier, and demonstrated the feasibility of the program. The AMS-02 was then built and flown, and is still on the International Space Station collecting data. AMS-02 has dimensions of $3 \times 4 \times 5$ meters, and weighs 7.5 tons. The detectors include a Transition Radiation Detector, an Electromagnetic Calorimeter, a Silicon Tracker, a Time of Flight counter, and a Ring Imaging Cherenkov counter system; these add up to about 300,000 channels of detectors. The data from the detectors are processed by 650 on-board computers. A good description of the AMS-02 device is in the Physical Review Letters paper [1]. It should be noted that the AMS-02 international collaboration includes over a hundred physicists, who are all coauthors on these publications, so - in references to these papers - only Ting's name (as the program spokesman) is cited.

It may be noted that, although Ting's home institution is M.I.T., Ting has long, close attachments to CERN (in Geneva, Switzerland), as former spokesman for the L3 experiment at the CERN electron-positron collider, LEP. Hence CERN has a building on their Prevessin cite which is occupied by AMS, and in which the data collection and analysis are located.

Attached here, as Figs. 1, 2, 3, and 4, are graphs of data from AMS-02: the positron/electron+positron fraction, the proton spectrum, the proton - anti-proton ratio and the iron nuclei spectrum. The published papers presenting these and other data are cited in references [2-5].

\section{References}

[1] S. C. C. Ting, Phys. Rev. Letters 110, 141102 (2013)

[2] S. C. C. Ting, Phys. Rev. Letters 113, 121102 (2014)

[3] S. C. C. Ting, Phys. Rev. Letters 113, 221102 (2014)

[4] S. C. C. Ting, Phys. Rev. Letters 114, 171103 (2015)

[5] S. C. C. Ting, Phys. Rev. Letters 117, 091103 (2016) 